\title{
The triple F knot: a sliding-locking arthroscopic knot
}

\author{
Filippo Spiezia ${ }^{1}$ \\ Edoardo Franceschetti ${ }^{2}$ \\ Francesco Franceschi ${ }^{2}$ \\ Nicola Maffulli ${ }^{3,4}$ \\ Francesco Oliva 5
}

1 Azienda Ospedaliera Regionale, San Carlo, Potenza, Italy

2 Department of Orthopaedic and Trauma Surgery, Campus Biomedico University, Rome, Italy

3 Department of Orthopaedics and Traumatology, Azienda Ospedaliera San Giovanni di Dio e Ruggi d'Aragona, University of Salerno, Salerno, Italy

4 Centre for Sports and Exercise Medicine Barts and The London School of Medicine and Dentistry, Mile End Hospital, London, England

5 Department of Orthopaedics and Traumatology, University of Rome "Tor Vergata", Rome, Italy

\section{Corresponding author:}

Nicola Maffulli

Department of Musculoskeletal Disorders

School of Medicine and Surgery

University of Salerno,

Mary University of London

Barts and the London School of Medicine and

Dentistry

Centre for Sports and Exercise Medicine

Mile End Hospital 275 Bancroft Road

E1 4DG, London, England, UK

Tel.: +447989358279

E-mail: n.maffulli@qmul.ac.uk

\section{Summary}

Introduction: Arthroscopic knot tying is a necessary skill for arthroscopic surgeons. Different arthroscopic knot configurations are available, and young surgeons may find it difficult to choose which one is best suited to them.

Methods: We describe a simple technique for a sliding locking arthoscopic knot, which is fast to execute, and easy to perform and to teach to young surgeons.

KEY WORDS: arthroscopic knot tying, arthroscopic technique, rotator cuff repair, suture.

\section{Introduction}

Arthroscopic knot tying is a necessary but challenging skill for arthroscopic surgeons to master. Even though advances in knotless technology have been made, it is still recommended that surgeons are fully conversant with at least one self sliding knot.

Several different sliding and non sliding, locking and non locking knot configurations have been presented. The Triple $\mathrm{F}$ knot is a fast, simple, and easy to teach and to perform sliding-locking knot for arthroscopic rotator cuff repair, using only three fingers of one hand.

\section{Materials and methods}

\section{Technique}

The Triple F technique for arthroscopic knot tying starts when both ends of the suture, passed through the tissue, are in the hands of the surgeon, having been retrieved through the arthroscopic cannula (Fig. 1). The "post" is the suture thread on which the other thread (non-post) will slide, and is the firm base on which the surgeon will concentrate. First of all, the post is wrapped twice around the index finger, so as to stabilize the post and maintain firm tension during the knot tying process. This is the first step of the technique (Fig. 2). In the second step, using the middle finger, the nonpost thread is locked and tensed by the middle finger (Fig. 3), three throws over both threads are carried out (Fig. $4 a, b)$.

The third step, using the ring finger, consists in producing a loop with the ring finger between the post and non-post thread, so that the end of non-post thread is brought inside this loop (Figs. 5, 6).

The knot is then tightened slightly pulling the nonpost (Figs. 7, 8). Finally, pulling on the post, the knot is advanced to the tissue sliding through the arthroscopic cannula (Fig. 9).

At this point, the surgeon can lock the knot pulling the non-post end, and the knot will not migrate backward (Fig. 10).

If desired, the knot can be further secured with alternating half hitches, to lock the configuration.

\section{Discussion}

Different configurations of arthroscopic sliding knots have been described ${ }^{1-5}$. The ideal sliding knot should 


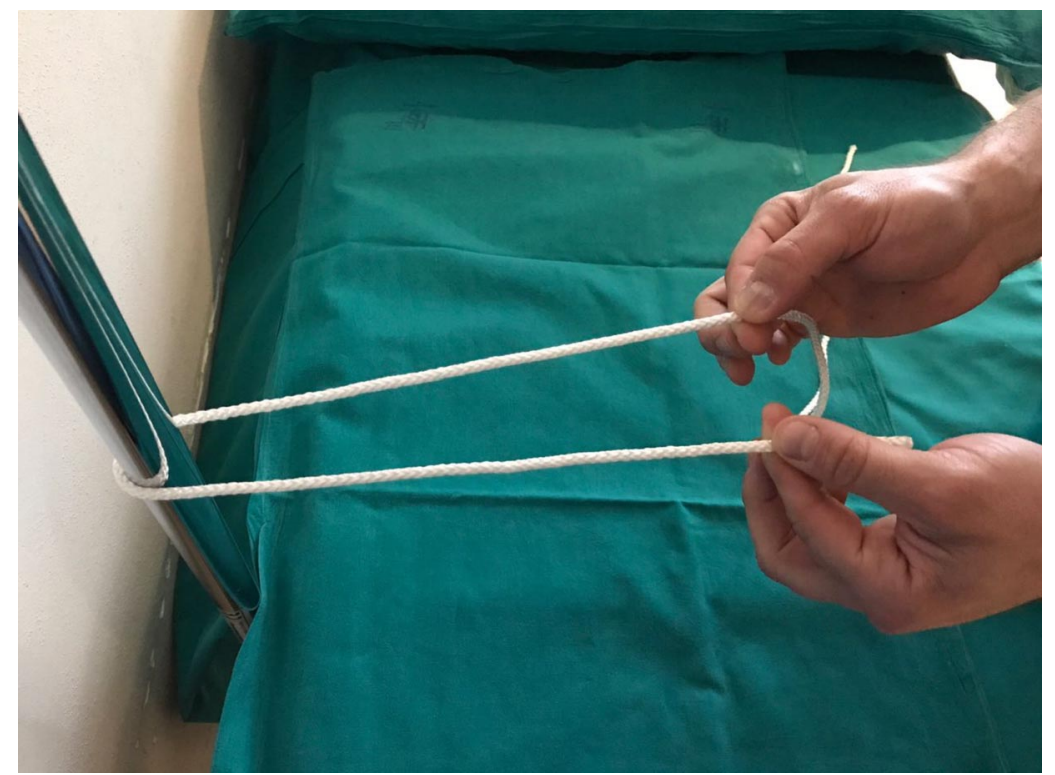

Figure 1. Starting position. The "post" is in the left hand.

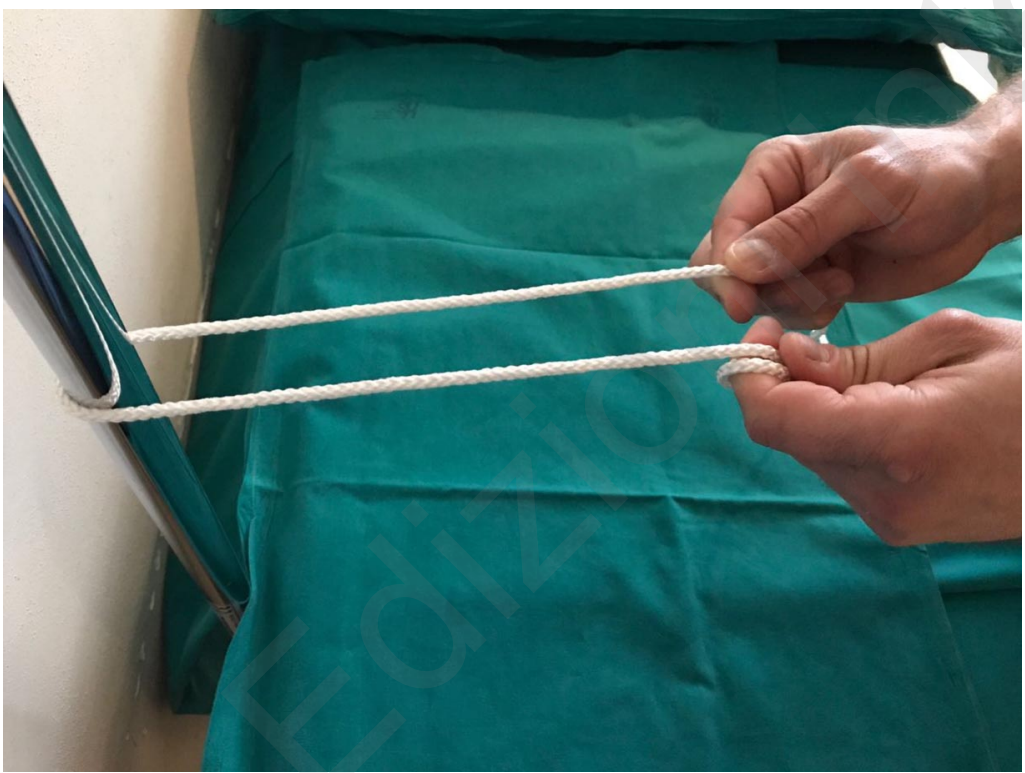

Figure 2. The post is wrapped twice around the index finger, to stabilize the post and maintain firm tension during the knot tying process.

be reliable, fast to perform, and easy to teach and memorize. It also needs to have a low profile, and has to slide through arthroscopic cannula slightly, maintaining soft tissue approximation, without loosening tension.

The Triple $F$ (triple finger) knot presents all these characteristics. However, it is mandatory to learn to knot in a non clinical environment, thus minimising the inevitable surgical learning curve.

When tying this knot, it is important to keep the post thread well tensed by the index finger (first finger step), and also to keep the same tension of both threads in the second finger step, using the middle finger. Obviously, the knot has to be tightened up to decrease the size of the knot before advancing through a cannula. A non tightened knot will not slide properly through the cannula, or it may lock while still in the cannula. It is also important to shorten the post thread, leaving enough suture to slide the knot through a cannula. The dressing of the knot before the sliding through the cannula has to be performed pulling gently the non-post thread, avoiding too much pulling, which will cause the premature locking of the knot.

This knot, in common with other locking and sliding knots, allows to maintain good suture tension and good contact between suture and tissue interface, which make it optimal for rotator cuff or instability repairs. The Triple $\mathrm{F}$ knot technique is fast to perform, and also easy to learn and teach. The technique has 


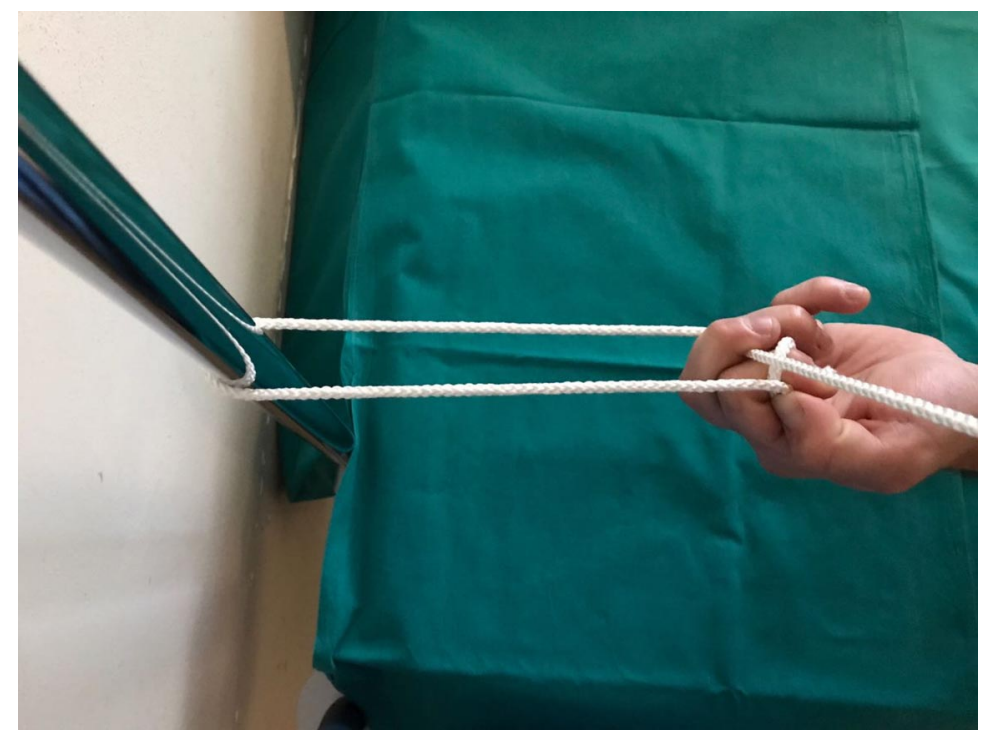

Figure 3. Using the middle finger, the nonpost thread is locked and tensioned by the middle finger.

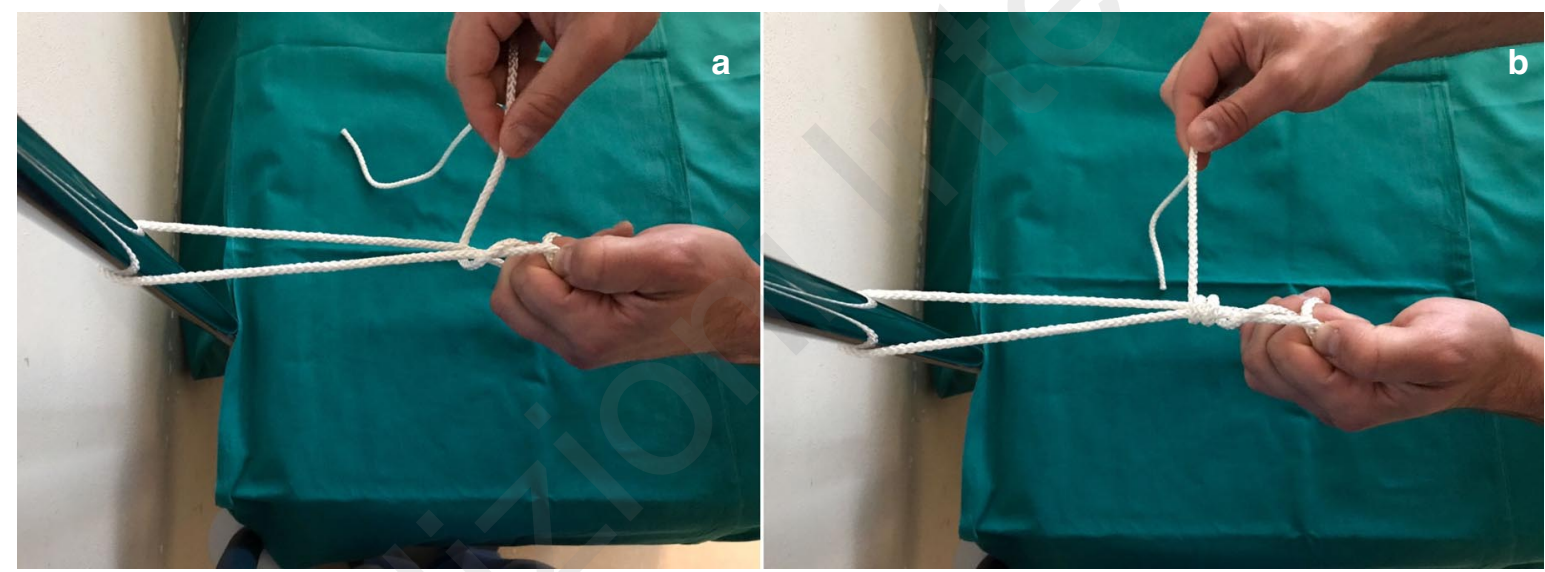

Figure 4 a. Three throws are performed over both threads. This figure shows the first throw. b. Three throws are performed over both threads. This figure shows the third throw.

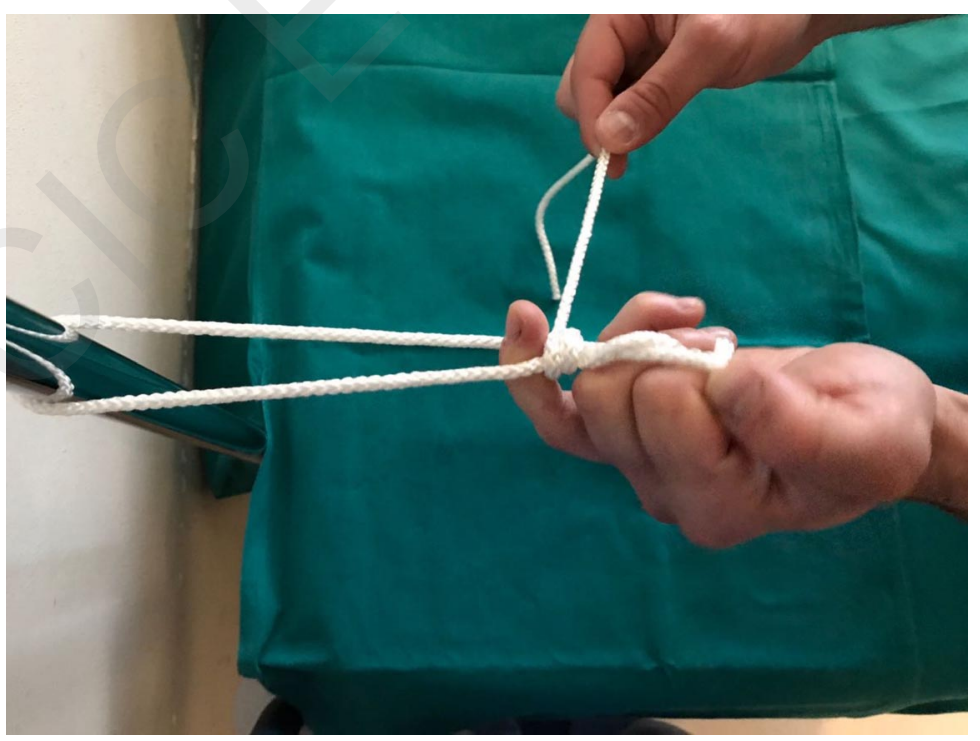

Figure 5. The ring finger is placed between the post and non-post thread. 

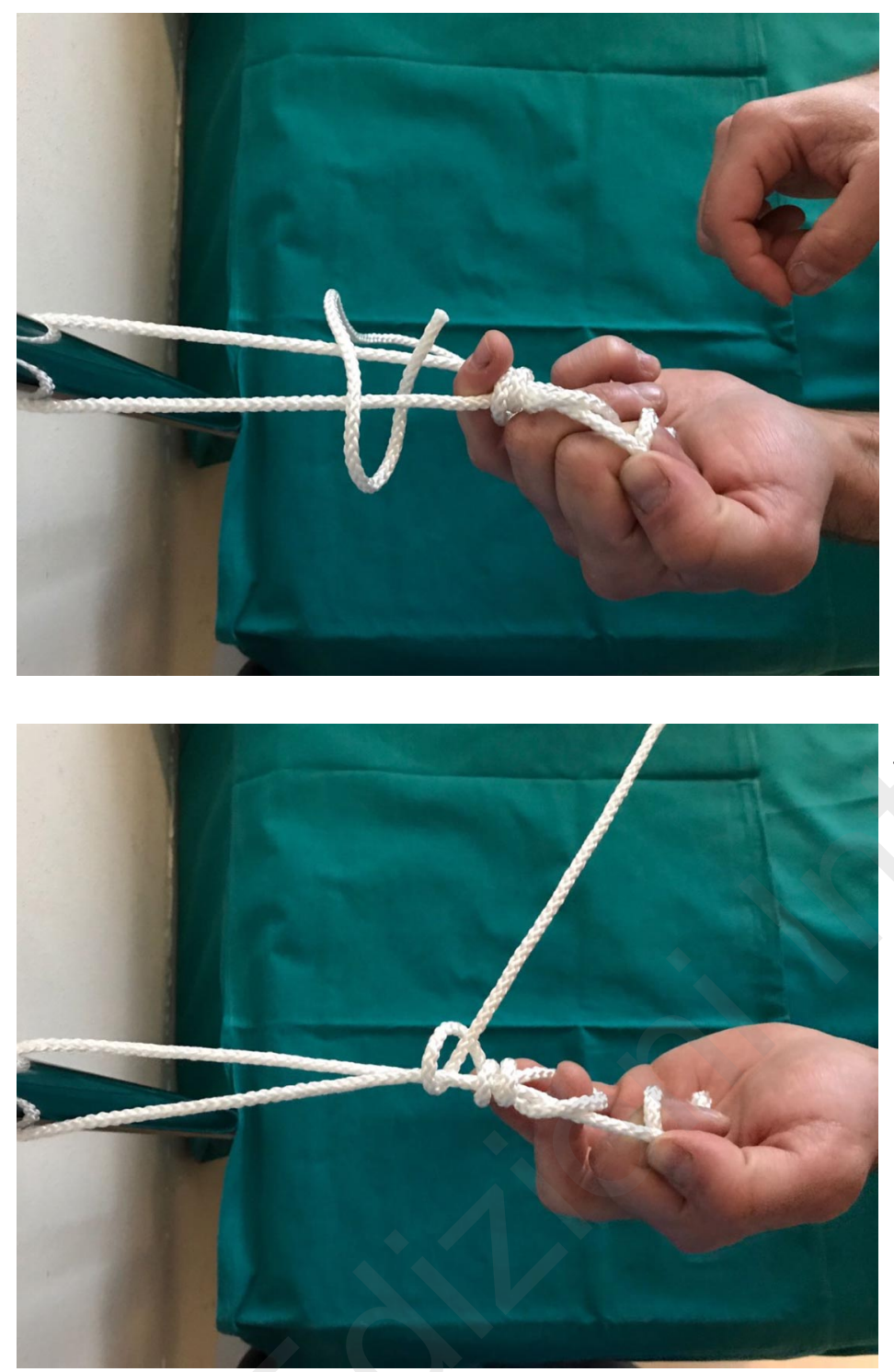

Figure 7. The knot is tightened gently pulling

the non-post.

Figure 6 . The end of the non-post thread is brought inside this loop.

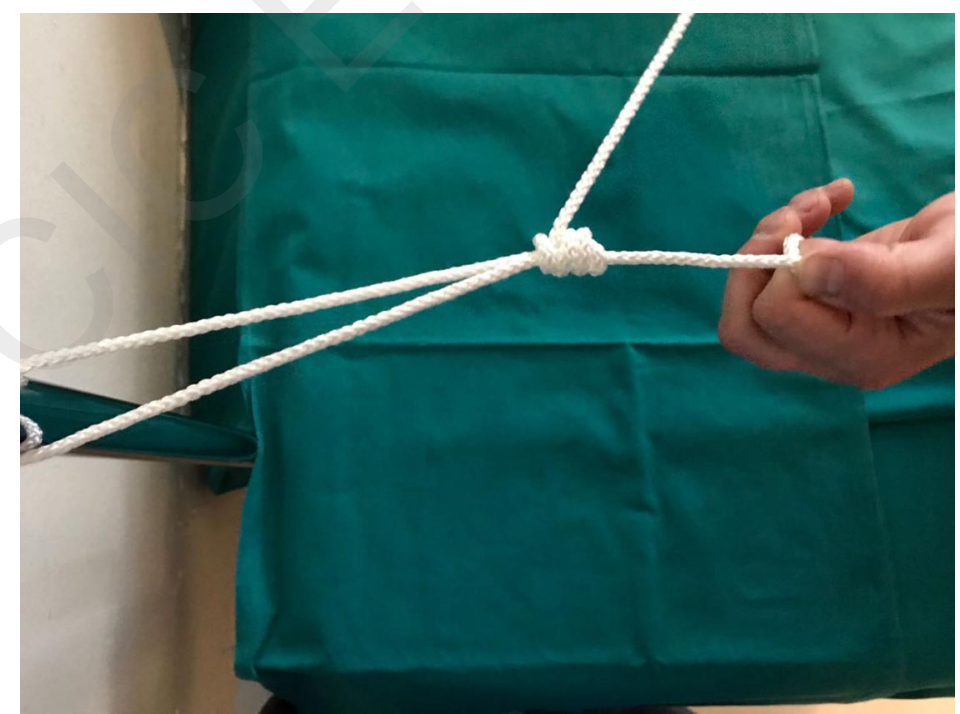

Figure 8. The knot is tightened and reaches its definitive configuration. 

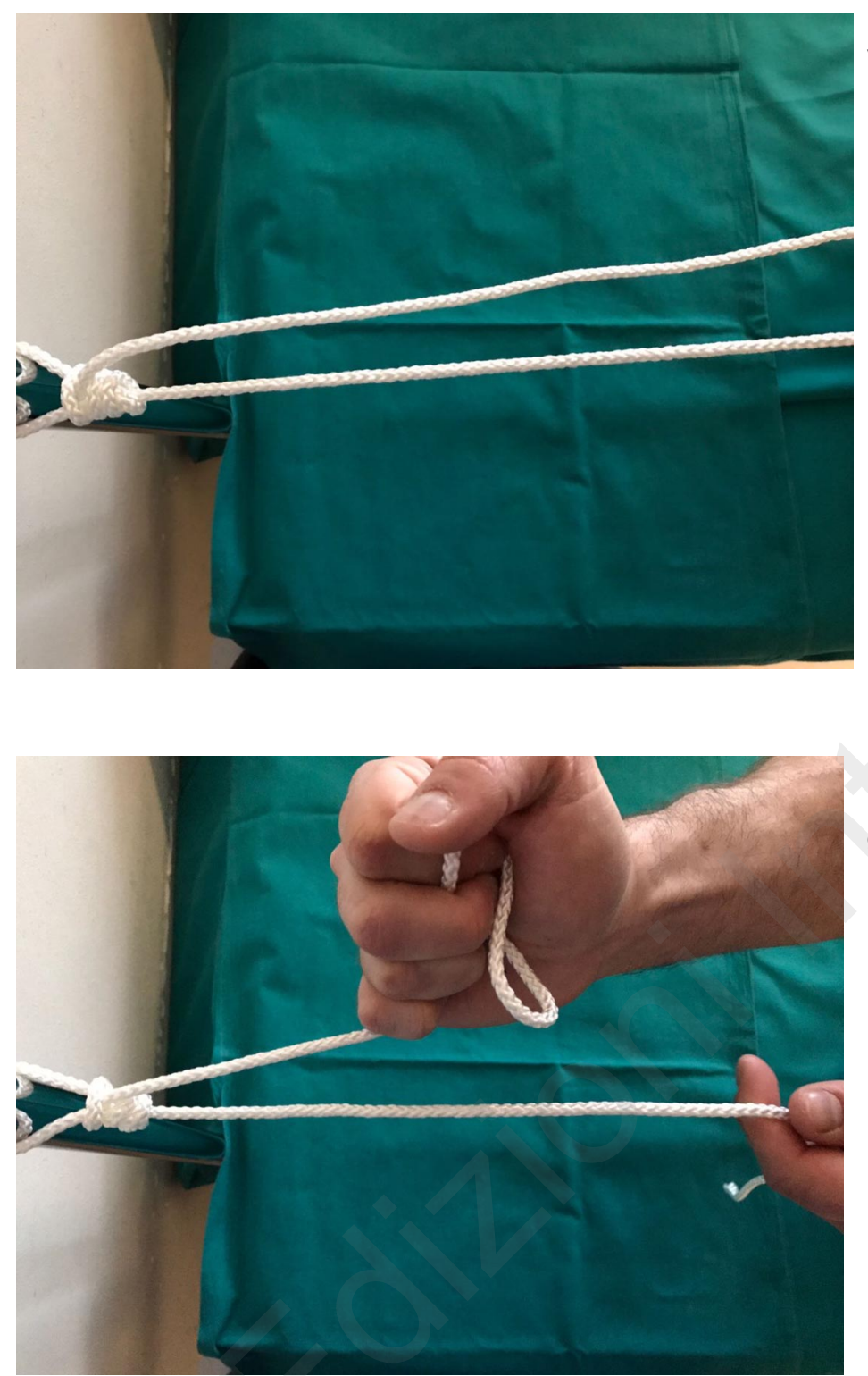

Figure 9. Pulling on the post, the knot is advanced to the tissue. some limitations. In common with all sliding knots, alternating half hitches should be added to increase the security of the construct. The sliding of the suture thread has to be carefully assessed before performing the Triple $\mathrm{F}$ knot, or the knot will never slide to the tissue. Finally, the sliding of the suture, while performing the Triple $F$ technique, may cause some friction, damaging the tissue.

\section{Compliance with ethical standards}

The Authors declare that this research was conducted following basic ethical aspects and international standards as required by the journal and recently update in ${ }^{6}$.
Figure 10. The knot is locked pulling the nonpost end with right hand. The knot will not migrate backward anymore.

\section{References}

1. Parada SA, Shaw KA, Eichinger JK, et al. The Wiese Knot: A Sliding-Locking Arthroscopic Knot. Arthrosc Tech. 2017;6:e21-e24.

2. Mochizuki Y, Hachisuka H, Natsu K, Kashiwagi K, Yasunaga Y, Ochi M. The HU knot: a new sliding knot for arthroscopic surgery. Arthroscopy. 2005;21:1014.

3. Kim SH, Ha KI. The SMC knot: a new slip knot with locking mechanism. Arthroscopy. 2000;16:563-565.

4. Nottage WM, Lieurance RK. Arthroscopic knot typing techniques. Arthroscopy. 1999;15:515-521.

5. De Beer JF, van Rooyen K, Boezaart AP. Nicky's knot: a new slip knot for arthroscopic surgery. Arthroscopy. 1998;14:109110.

6. Padulo J, Oliva F, Frizziero A, Maffulli N. Muscles, Ligaments and Tendons Journal - Basic principles and recommendations in clinical and field science research: 2016 update. MLTJ. 2016;6(1):1-5. 